\title{
Incidencia del estrés en la fibromialgia
}

Dra. Cristina Camacho F*

\section{SÍNTESIS}

La Fibromialgia (FM) se reconoce hoy como un síndrome de dolor en el que la depresión y el estrés son consecuencias. Síntomas de dolor, cansancio y alteraciones en el sueño varían en intensidad y frecuencia e interfieren en actividades diarias causando emociones y comportamientos negativos en la persona y alterando las relaciones familiares, sociales y laborales; llevando a múltiples situaciones de estrés que dificultan el control de la enfermedad. Por eso, el énfasis en ofrecer un tratamiento multidisciplinario a quien la padece a la vez que educar y asesorar al medio en general.

\section{ABSTRACT}

The Fibromyalgia (FM) it is recognized today like a pain syndrome in which the depression and the stress are consequences. Pain symptoms, tire and alterations in the dream vary in intensity and frequency and they interfere in daily activities causing emotions and negative behaviors in the person and altering the family, social and work relationships; taking to multiple stress situations that hinder the control of the illness. For that reason, the emphasis in offering a multidisciplinary treatment to those who suffer it and at the same time to educate and to advise the population in general.

* Psicóloga Clínica Colombiana de Reumatología, Salud Reinun. Profesora: Facultad de Fisioterapia, Corporación Universitaria lberoamericana. 


\section{INTRODUCCIÓN}

La fibromialgia (FM) significa dolor en los músculos y el tejido fibroso. Fue descrita inicialmente como un desorden inflamatorio(1). Hoy se le reconoce como un síndrome de dolor no psicosomático y que cuando están presentes el estrés y la depresión son éstos más la consecuencia del síndrome que su causa $(2,3$,$) . Se cree que$ el dolor de la FM se debe a microtraumas en los músculos poco ejercitados, pero estudios muestran que no necesariamente hay anormalidades en los músculos afectados (4), igualmente se atribuye a la mala calidad del sueño en el cual la persona no llega a tener un sueño profundo adecuado (5). Sin embargo, en la actualidad no se ha logrado llegar a conocer su etiología real, así que el diagnóstico se hace más a partir de su sintomatología.

La (FM) es una enfermedad reumática, reconocida por la Organización Mundial de la Salud, caracterizada por la presencia de puntos selectivos de dolor generalmente bilaterales y dolores musculares difusos; los cuales a veces comienzan en forma generalizada y en otros en un área determinada como el cuello, el hombro, la columna lumbar y desde ahí se extiende. Además del dolor la FM puede ocasionar rigidez generalizada sobre todo en la mañana al levantarse y el cansancio a lo largo del día. Las personas con FM presentan con frecuencia ansiedad, depresión, jaquecas, colón irritable, trastornos de la circulación y sueño de mala calidad. Es probable que la mala adaptación a las situaciones de estrés sea un rasgo importante en estas personas. Se han descrito casos en que la FM comienza después de períodos concretos de estrés como puede ser una enfermedad infecciosa, un accidente, la separación, el divorcio o problemas con los hijos; indicando que la respuesta a éstos desencadena altos niveles de estrés que activan la enfermedad en la persona predispuesta.

Teniendo en cuenta que la FM es una enfermedad crónica para la cual no existe curación actualmente, resulta importante la intervención multidisciplinaria (reumatólogos, terapeutas ocupacionales, físicos, fisiatra y psicólogos) para mejorar la calidad de vida de quienes la padecen (principalmente mujeres entre los $30 y$ 50 años).

Aunque no causa incapacidad irreversible por deformidad o destrucción articular, sí es incapacitante en cuanto dificulta la realización de actividades diarias puesto que el dolor y el cansancio síntomas principales- varian en intensidad y se presen- 
$\tan$ ante el ejercicio moderado, lo que hace que se evite y traiga como consecuencia que cada vez se hagan menos esfuerzos y se debilite la masa muscular y la tolerancia al ejercicio sea cada vez menor (6). La figura 1 muestra más claramente la relación de síntomas en la FM.

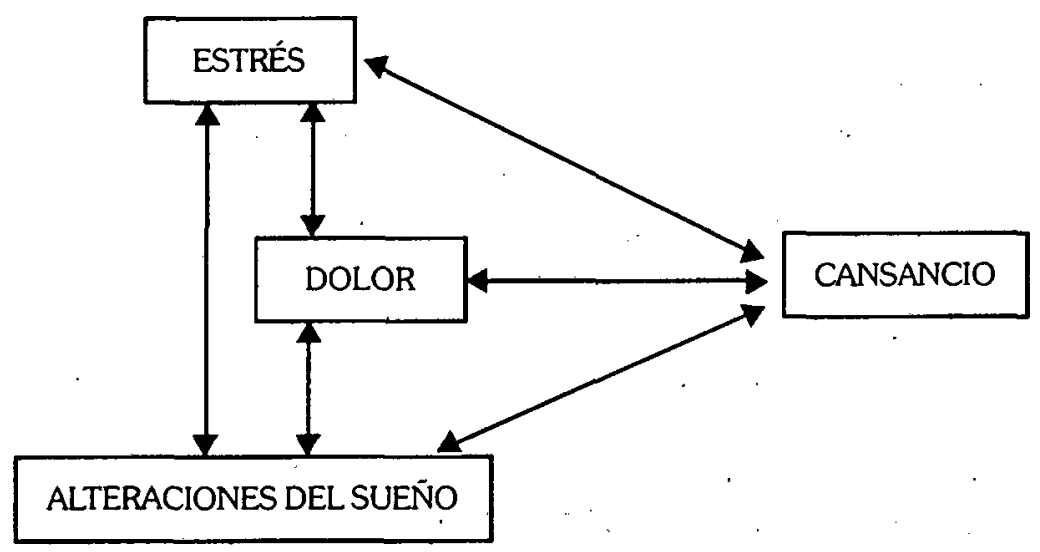

Fig. 1. Relación de los síntomas en la fibromialgia

Asociado a lo anterior están las implicaciones desde el punto de vista psicológico. En la medida en que los síntomas ya anotados interfieren con las actividades diarias de la persona con FM estas se encuentran con una pérdida de reforzadores, es decir, actividades que anteriormente realizaban sin dificultad y les eran gratificantes (por ejemplo caminar, ir de compras, practicar deportes, realizar trabajos manuales, bailar, participar en reuniones sociales entre otros), ya no lo son igual; de hecho, es frecuente encontrar que la persona se aísla de otros, se torna irritable, pierde la respuesta de alegría, tiene una baja motivación y se ve alterada negativamente su auto estima. Lo anterior puede explicar el porqué de la depresión moderada que se encuentra la mayoría de las veces en éstas personas.

Relacionado con lo ya descrito está la manifestación de la ansiedad en el estrés. La persona intenta seguir enfrentando situaciones estresantes laborales, familiares y sociales, a pesar de los síntomas de la FM "como si ésta no estuviera presente"; ello conlleva a un aumento del estrés cuando observa que cada vez su rendimiento físico $e$ intelectual es menor, debido principalmente al dolor, el cansancio y los problemas de sueño; así entonces, la persona comienza a dudar de sus capacida- 
des, se angustia y teme que la enfermedad termine incapacitándola severamente. Estas creencias explican la razón por la que la persona con FM intenta una y otra vez obligarse a seguir en sus $A V D$ y en sus $A B C$ como siempre lo ha hecho, rechazando la enfermedad, rechazando los síntomas y muchas veces un tratamiento integral que le ayude a controlar adecuadamente los síntomas.

Además la falta de información sobre esta enfermedad y el hecho de que no sea fácilmente observable por otros conduce con frecuencia a que la persona con FM se sienta aislada, sola y desorientada para enfrentarla. El especialista (reumatólogo) requiere de una buena historia clínica y un examen físico completo para llegar a un diagnóstico apropiado; muchas veces se presenta la FM secundaria, es decir, manifestaciones clínicas de la FM al lado de manifestaciones de otra enfermedad reumatológica como puede ser en lupus, artritis reumatoide y la osteoartritis lo cual hace más difícil el diagnóstico acertado y esto tiene un efecto emocional muy negativo en la persona, pues propicia cambios frecuentes de médico y la tendencia a ser sobre estudiados con exámenes auxiliares costosos e innecesarios que aumentan la ansiedad de la persona y mantienen la idea equivocada de que "no tienen nada mal y todo está es en su cabeza". (7) Esto lleva a la persona a mostrarse defensiva ante las evaluaciones $e$ intervenciones del equipo multidisciplinario; con la consecuencia de la baja adherencia a las recomendaciones y la demora en observar resultados positivos del tratamiento. Sumado a la actitud de la persona con FM está la del medio en general. Familiares, amigos y compañeros de trabajo pueden restar importancia a la enfermedad y los síntomas; exigiendo de la persona un buen desempeño constante en las actividades diarias, lo cual aumenta el estrés y la siente confundida, abrumada y temerosa para hacer frente a su enfermedad. Es común que los familiares duden de la veracidad de los síntomas que manifiesta la persona, puesto que el médico les dice que los análisis y las radiografías son normales, a pesar de que la queja común de la persona con FM es "me duele todo".

Con frecuencia los síntomas varían en relación con la hora del día, el nivel de actividad, los cambios climáticos, la falta de sueño y el estrés; lo cual lleva a que la persona tenga baja tolerancia al esfuerzo físico y que se sienta agotada, sin energía. Así es comprensible que se vean afectadas las relaciones interpersonales en general y laborales er، particular.

Familiares y amigos la consideran perezosa y falta de motivación y la recriminan por su bajo rendimiento en las diversas actividades y en el cumplimiento de respon- 
sabilidades, con la agravante de que la excluyen de actividades sociales y laborales logrando que la persona se sienta afectada en su auto estima, pues comienza a creer que es una inútil que no puede responder a las expectativas de otros y duda de su propio valor como persona. En las relaciones familiares se observa el disgusto de sus miembros porque tienen que asumir tareas de la persona con FM aparte de sus propias obligaciones. Además las actividades recreativas y sociales se ven alteradas, pues igualque ocurre con otros problemas crónicos en los que el dolor está presente, en la FM la persona se ve obligada a modificar el ritmo y la cantidad de actividad que realiza diariamente dependiendo de la presencia y severidad de los síntomas; así los familiares no saben realmente si pueden "contar con ella o no" en las actividades, lo cual altera las relaciones sino existe una buena comunicación y un apoyo social positivo por parte de todos.

Tampoco los amigos comprenden los cambios en la sintomatología y en el ánimo de la persona que los padece; el cansancio y el dolor son considerados muchas veces como quejas para llamar la atención o para lograr que otros hagan lo que la persona quiere. Entonces, comienzan a alejarse de ella o es ella quien se aísla de su grupo social para evitar el rechazo.

En el ámbito lab.oral las relaciones también se ven deterioradas con frecuencia. Dado que el rendimiento físico está disminuido y la concentración baja sobre todo en actividades que impliquen esfuerzo intelectual continuado; jefes, compañeros de trabajo y subalternos se quejan porque la persona no rinde o no es lo suficientemente competitiva para las exigencias del medio y atrasa el trabajo de otros o los obliga a asumir sus responsabilidades sobrecargándolos. Esta situación genera conflicto para uno y para otros. La persona con FM asume una actitud bien sea de exigirse más a pesar de los síntomas y esto empeora su condición de salud, o bien adopta una conducta de víctima de las circunstancias, creando en los demás sentimientos y conductas de hostilidad. En varios casos la situación puede ser tan tensa que obliga a un retiro temporal o definitivo y en el mejor de los casos a una reubicación laboral que permita a la persona trabajar sin exigencias tan elevadas que afecten negativamente su salud.

Frente a lo ya descrito es más fácil entender que la persona con FM enfrenta múltiples situaciones de estrés en su vida diaria que dificultan más el control de la enfermedad; por ello se hace especial énfasis en que el tratamiento no sólo brinde la medicación necesaria, sino que también enseñe a la persona a ejecutar sus activi- 
dades de forma diferente y le enseñe a afrontar el estrés en forma adecuada. Igualmente se debe tener en cuenta el educar y asesorar al medio en general especialmente familiary laboral sobre ésta enfermedad reumática y la forma de brindar un apoyo efectivo.

Con lo anterior se intenta tener una perspectiva más clara de la necesidad de comprender, informar y tratar a las personas que presentan FM. En la búsqueda de una intervención efectiva es necesario considerar a la persona siempre como un ser integral en el que sus aspectos biológicos, psicológicos y sociales están afectados. De ahí, la importancia de ofrecerles un tratamiento multidisciplinario que incluya además de la medicación el ejercicio dirigido, los masajes, la adquisición de hábitos posturales adecuados en las diversas actividades y trabajos, el aprendizaje de estrategias cognitivo-conductuales y la nutrición adecuada. Todo ello para proporcionar a la persona herramientas para controlar los síntomas y afrontar mejor la FM. Es importante que se realice investigación en éste campo logrando avances en el conocimiento de los mecanismos físicos y químicos que se producen en el cuerpo en respuesta a estímulos externos que provocan estrés y avanzar cada día en la búsqueda de tratamientos multidisciplinarios más eficaces.

\section{REFERENCIAS}

1. GOWERS, W.R.Lumbago its lessons and analogues. Br Med J. 1:117, 1904.

2. GOLDENBERG, D.L. Psychological symptoms and psychiatric diagnosis in patients with Fibromyalgia. J. Rheumatol. 16 (suppl 19): 127, 1989.

3. YUNUS, M.B. et al. Relatioship of clinical features with psychological status in primary fibromyalgia. Arthritis Rheum 34(1): 15-21, 1991.

4. SCHRODER, H.D. et al. Muscle biopsy in fibromyalgia. J. Musculoskel Pain 1(3/ 4): 165, 1993.

5. MOLDOSFKY, H.D. A chronobiologic theory of fibromyalgia. J. Muculoskel Pain $1(3 / 4): 49,1993$.

6. TEVAR, J.P. Fibromialgia: guía de información aspectos clínicos. 3-4,1997*

7. ALARCÓN, G.S.; BRADLEY, L.A. Avances en fibromialgia. 4,1999*

* Artículos del Internet. 